\title{
ON THE MINIMAL GRADED FREE RESOLUTION OF POWERS OF LEXSEGMENT IDEALS
}

\author{
Anda Olteanu
}

\begin{abstract}
We consider powers of lexsegment ideals with a linear resolution (equivalently, with linear quotients) which are not completely lexsegment ideals. We give a complete description of their minimal graded free resolution.
\end{abstract}

\section{Introduction}

Let $S=K\left[x_{1}, \ldots, x_{n}\right]$ be the polynomial ring in $n$ variables over a field $K$ and $<_{\text {lex }}$ the lexicographical order with respect to $x_{1}>_{\text {lex }}>\cdots>_{\text {lex }} x_{n}$. Fix an integer $d \geq 2$ and let $u$ and $v$ be two monomials of degree $d$ in $S$ such that $u>_{\text {lex }} v$. The lexsegment ideal determined by the monomials $u$ and $v$, $(\mathcal{L}(u, v))$, is the monomial ideal generated by all the monomials $w$ in $S$ of degree $d$ which have the property that $u>_{\text {lex }} w>_{\text {lex }} v$.

Defined by H. Hulett and H. M. Martin [8], lexsegment ideals have been studied in several papers $[1,4,5,6,9]$. Their properties such as being Gotzmann, normally torsion-free or sequentially Cohen-Macaulay have been completely characterized $[9,10,11]$. All the characterizations are in terms of the numerical data of the monomials that determine the lexsegment.

It is known that any ideal with linear quotients generated in one degree has a linear resolution, but the converse does not hold (see, for instance, [3, Lemma 1 and Example 4.2]). In [5, Theorem 1.2 and Theorem 2.1], it is proved that these two notions are equivalent for the class of lexsegment ideals. Moreover, for the case of completely lexsegment ideals with linear quotients, the minimal graded free resolution can be described. It is natural to ask whether the powers of an ideal with linear quotients have again linear quotients. Conca's example shows

Received June 19, 2012; Revised August 7, 2012.

2010 Mathematics Subject Classification. Primary 13D02; Secondary 13C15, 13H10, $13 \mathrm{P} 10$. ideal.

Key words and phrases. lexsegment ideals, linear resolution, linear quotients, monomial

The author was supported by the CNCS-UEFISCDI project PN II-RU PD 23/06.08.2010 and by the strategic grant POSDRU/89/1.5/S/58852, Project "Postdoctoral program for training scientific researchers" co-financed by the European Social Fund within the Sectorial Operational Program Human Resources Development 2007-2013. 
that this is not true in general [2, Example 3.2], but for lexsegment ideals, this property is preserved by their powers [6, Theorem 2.10 and Corollary 3.9].

We will consider powers of lexsegment ideals with a linear resolution which are not completely lexsegment ideal and we describe their minimal graded free resolution by proving that their decomposition function is regular and using the result of J. Herzog and Y. Takayama for this case [7]. In this way, the minimal graded free resolution of lexsegment ideals with linear quotients is completely described.

The paper is organized in three sections. In the first section, we define all the notations and the terminologies and we recall some known results which will play a key role in the proofs.

In the second section, we consider powers of a lexsegment ideal $I$ with linear quotients which is not a completely lexsegment ideal. We describe the decomposition function associated with the increasing reverse lexicographical order and we show that this is regular. By using the results of J. Herzog and Y. Takayama [7], we write the minimal graded free resolution of $I^{k}$ for all $k \geq 1$.

In the last section, we consider an example in order to illustrate the results.

\section{Preliminaries}

Let $S=K\left[x_{1}, \ldots, x_{n}\right]$ be the polynomial ring in $n$ variables over a field $K$ and we fix the lexicographical order, $<_{l e x}$, on $S$ with respect to the order of the variables $x_{1}>_{\text {lex }}>\cdots>_{\text {lex }} x_{n}$. For a monomial $m=x_{1}^{a_{1}} \cdots x_{n}^{a_{n}}$, we denote by $\nu_{i}(m)$ the exponent of the variable $x_{i}$ in the monomial $m$, that is, $\nu_{i}(m)=a_{i}$. The set $\operatorname{supp}(m)=\left\{i: \nu_{i}(m) \neq 0\right\}$ is called the support of the monomial $m$. Let us denote $\min (m):=\min (\operatorname{supp}(m))$ and $\max (m):=\max (\operatorname{supp}(m))$. If $I$ is a monomial ideal in $S$, then $G(I)$ will be the set of its minimal monomial generators.

For $d \geq 2$ an integer, we denote by $\mathcal{M}_{d}(S)$ the set of all monomials of degree $d$ in $S$. Let $u, v \in \mathcal{M}_{d}(S)$ be two monomials such that $u>_{\text {lex }} v$. The set

$$
\mathcal{L}(u, v)=\left\{w \in \mathcal{M}_{d}(S): u>_{\text {lex }} w>_{\text {lex }} v\right\}
$$

is called the lexsegment set determined by the monomials $u$ and $v$. A lexsegment ideal is a monomial ideal generated by a lexsegment set. An important notion in the study of the lexsegment ideals is the shadow of a set of monomials. For a set of monomials $T \subseteq S$, one may define its shadow as being the set $\operatorname{Shad}(T)=\left\{x_{i} w: 1 \leq i \leq n, w \in T\right\}$. Moreover, the $i$ th shadow is recursively defined as $\operatorname{Shad}^{i}(T)=\operatorname{Shad}^{i-1}(\operatorname{Shad}(T))$.

A lexsegment set is called a completely lexsegment set if all the iterated shadows are again lexsegment sets. An ideal generated by a completely lexsegment set is called a completely lexsegment ideal.

In [7, pg. 278], the class of ideals with linear quotients is considered. 
Definition 1.1 ([7]). A monomial ideal $I \subseteq S$ has linear quotients if there exists an ordering of its minimal monomial generators $m_{1}, \ldots, m_{r}$ such that the ideal $\left(m_{1}, \ldots, m_{i-1}\right):\left(m_{i}\right)$ is generated by a set of variables for all $i \geq 2$.

If $I$ is a monomial ideal which has linear quotients with respect to the sequence $m_{1}, \ldots, m_{r}$, then one may consider the sets

$$
\operatorname{set}\left(m_{i}\right)=\left\{j: x_{j} \in\left(m_{1}, \ldots, m_{i-1}\right):\left(m_{i}\right)\right\}
$$

for all $i \geq 2$.

The following result collects known results on lexsegment ideals.

Theorem $1.2([1,5,6])$. Let $u=x_{1}^{a_{1}} \cdots x_{n}^{a_{n}}$ with $a_{1}>0$ and $v=x_{1}^{b_{1}} \cdots x_{n}^{b_{n}}$ be monomials of degree $d$ with $u \geq_{\text {lex }} v$ and let $I=(\mathcal{L}(u, v))$ be a lexsegment ideal. Then the following statements are equivalent:

(1) I has a linear resolution.

(2) I has linear quotients.

(3) All the powers of I have linear quotients.

(4) All the powers of I have a linear resolution.

If we restrict to the case of lexsegment ideals which are not completely lexsegment ideals, we have the following result which combines $[1$, Theorem 2.4], [5, Theorem 2.1], and [6, Corollary 3.9]:

Theorem 1.3. Let $u=x_{1}^{a_{1}} \cdots x_{n}^{a_{n}}$ with $a_{1}>0$ and $v=x_{2}^{b_{2}} \cdots x_{n}^{b_{n}}$ be monomials of degree $d$ with $u \geq_{\text {lex }} v$, and let $I=(\mathcal{L}(u, v))$ be a lexsegment ideal which is not completely lexsegment. Then the following statements are equivalent:

(1) $u$ and $v$ have the following form:

$$
u=x_{1} x_{l+1}^{a_{l+1}} \cdots x_{n}^{a_{n}} \text { and } v=x_{l} x_{n}^{d-1}
$$

for some $l, 2 \leq l \leq n-1$.

(2) I has a linear resolution.

(3) I has linear quotients.

(4) All the powers of I have linear quotients.

(5) All the powers of I have a linear resolution.

The order of the minimal monomial generators for which $I^{k}$ has linear quotients for all $k \geq 1$, where $I$ is a lexsegment ideal with a linear resolution which is not completely lexsegment ideal, is the increasing reverse lexicographical order. We recall that $m_{1}<_{\text {revlex }} m_{2}$ if there is some $s, 1 \leq s \leq n$, such that $\nu_{i}\left(m_{1}\right)=\nu_{i}\left(m_{2}\right)$ for all $i \geq s$ and $\nu_{s}\left(m_{1}\right)>\nu_{s}\left(m_{2}\right)$.

Remark 1.4. Let $u, v \in \mathcal{M}_{d}$ be two monomials, $u \geq_{\text {lex }} v$, and $I=(\mathcal{L}(u, v))$ be the corresponding lexsegment ideal. Note that we may always assume $x_{1} \mid u$ and $x_{1} \nmid v$. Indeed, if $x_{1} \mid v$, then we denote $u=x_{1}^{a_{1}} \cdots x_{n}^{a_{n}}$ and $v=x_{1}^{b_{1}} \cdots x_{n}^{b_{n}}$ with $a_{1} \geq b_{1}>0$. If $a_{1}=b_{1}$, then $I=(\mathcal{L}(u, v))$ is isomorphic, as an $S$-module, to the ideal generated by the lexsegment $\mathcal{L}\left(u / x_{1}^{a_{1}}, v / x_{1}^{b_{1}}\right)$ of degree $d-a_{1}$. This lexsegment may be studied in the polynomial ring in a smaller number of 
variables. If $a_{1}>b_{1}$, then $I=(\mathcal{L}(u, v))$ and $\left(\mathcal{L}\left(u / x_{1}^{b_{1}}, v / x_{1}^{b_{1}}\right)\right)$ are isomorphic as $S$-modules and we have $\nu_{1}\left(u / x_{1}^{b_{1}}\right)>1$ and $\nu_{1}\left(v / x_{1}^{b_{1}}\right)=0$. Therefore, we will always assume that $x_{1} \mid u$ and $x_{1} \nmid v$.

\section{Powers of lexsegment ideals with a linear resolution which are not completely lexsegment ideals}

In the sequel, we show that all the powers of lexsegment ideals with a linear resolution which are not completely lexsegment ideals have a regular decomposition function with respect to the increasing reverse lexicographical order. For two monomials $u, v$ of degree $d$, we denote by $\mathcal{L}(u, v)$ the corresponding lexsegment ideal. We will consider only the case when $x_{1} \mid u$ and $x_{1} \nmid v$.

By using Theorem 1.3, we will assume that $u$ and $v$ are monomials of degree $d \geq 2$ such that $I=(\mathcal{L}(u, v))$ is a lexsegment ideal which is not a completely lexsegment ideal, and that $u$ and $v$ have the following form:

$$
u=x_{1} x_{l+1}^{a_{l+1}} \cdots x_{n}^{a_{n}} \text { and } v=x_{l} x_{n}^{d-1}
$$

for some $l, 2 \leq l \leq n-1$.

For a lexsegment $\mathcal{L}(u, v)$, we assume that the elements are ordered by the increasing reverse lexicographical order. We denote by $I=(\mathcal{L}(u, v))$ the lexsegment ideal, and by $I_{<_{\text {revlex }} w}^{k}$, the ideal generated by all the monomials $z \in G\left(I^{k}\right)$ with $z<_{\text {revlex }} w . I_{\leq_{\text {revlex }} w}^{k}$ will be the ideal generated by all the monomials $z \in G\left(I^{k}\right)$ with $z \leq_{\text {revlex }} w$.

In order to describe the decomposition function, we need some preparatory results.

Lemma 2.1. Let $I=(\mathcal{L}(u, v)) \subset S$ be a lexsegment ideal with a linear resolution which is not a completely lexsegment ideal and $m \in G\left(I^{k}\right)$ a monomial. If $s \in \operatorname{set}(m)$, then $s>\min (m)$.

Proof. Since $m \in G\left(I^{k}\right)$ and $s \in \operatorname{set}(m)$, there exists a monomial $w \in G\left(I^{k}\right)$, $w<_{\text {revlex }} m$ such that $x_{s} m=x_{t} w$ for some $t, 1 \leq t \leq n$. Obviously, $m \neq w$ implies $s \neq t$ and $x_{t} \mid m$. Moreover, $w=x_{s} m / x_{t} m<_{\text {revlex }} m$ gives $s>t$. The statement follows since $x_{t} \mid m$ implies $t \geq \min (m)$.

One may note that, once we fix an integer $l, 2 \leq l \leq n-1$, a monomial $m \in S$ may be uniquely written as $m=\bar{m} \tilde{m}$ with $\bar{m} \in K\left[x_{1}, \ldots, x_{l}\right]$ and $\tilde{m} \in K\left[x_{l+1}, \ldots, x_{n}\right]$. In particular, we have $\max (\bar{m}) \leq l<\min (\tilde{m})$. On the set of all monomials of degree $k d$ in $S, \mathcal{M}_{k d}(S)$, we define the order $\prec$ as follows: for $m_{1}, m_{2} \in \mathcal{M}_{k d}(S)$, we say that $m_{1} \prec m_{2}$ if $\operatorname{deg}\left(\overline{m_{1}}\right)<\operatorname{deg}\left(\overline{m_{2}}\right)$ or $\operatorname{deg}\left(\overline{m_{1}}\right)=\operatorname{deg}\left(\overline{m_{2}}\right)$ and $m_{1}<_{\text {lex }} m_{2}$.

If $I=(\mathcal{L}(u, v))$ with $x_{1} \mid u$ and $x_{1} \nmid v$ is a lexsegment ideal with a linear resolution which is not a completely lexsegment ideal, then $u=x_{1} x_{l+1}^{a_{l}+1} \cdots x_{n}^{a_{n}}$ and $v=x_{l} x_{n}^{d-1}$ for some integer $l, 2 \leq l \leq n-1$. Therefore, through this paper, we will assume that the fixed integer which will be used in the order $\prec$ is $l$. 
Remark 2.2. If $m \in G\left(I^{k}\right)$, then $\operatorname{deg}(\bar{m}) \geq k$ since $u=x_{1} x_{l+1}^{a_{l+1}} \cdots x_{n}^{a_{n}}$ and $v=$ $x_{l} x_{n}^{d-1}$ for some $l, 2 \leq l \leq n-1$.

Lemma 2.3. Let $I=(\mathcal{L}(u, v)) \subset S$ be a lexsegment ideal with a linear resolution which is not a completely lexsegment ideal and $m \in G\left(I^{k}\right)$ a monomial. If $s \in \operatorname{set}(m)$ and $x_{s} m / x_{\min (m)} \prec v^{k}$, then $s>\min (\tilde{m})$.

Proof. By the hypothesis, we have $x_{s} m / x_{\min (m)} \prec v^{k}$. Writing $m$ as $m=\bar{m} \tilde{m}$, we get that the only possible case is when $\operatorname{deg}\left(\overline{x_{s} m / x_{\min (m)}}\right)<\operatorname{deg}\left(\overline{v^{k}}\right)=$ $\operatorname{deg}\left(\overline{x_{l}^{k} x_{n}^{k(d-1)}}\right)=k$. Indeed, if we assume that $\operatorname{deg}\left(\overline{x_{s} m / x_{\min (m)}}\right)=\operatorname{deg}\left(\overline{v^{k}}\right)=$ $k$, then $x_{s} m / x_{\min (m)}<_{l e x} v^{k}=x_{l}^{k} x_{n}^{k(d-1)}$. In particular, $x_{s} m / x_{\min (m)} \leq_{l e x}$ $x_{l}^{k-1} x_{l+1}^{k(d-1)+1}$ since

$$
x_{l}^{k-1} x_{l+1}^{k(d-1)+1}=\max _{l e x}\left\{w \in \mathcal{M}_{k d}(S): w<_{l e x} x_{l}^{k} x_{n}^{k(d-1)}\right\},
$$

a contradiction. Therefore, we have $\operatorname{deg}\left(\overline{x_{s} m / x_{\min (m)}}\right)<k$ which implies that $\operatorname{deg}(\bar{m})=k$ and $s>l$.

Since $s \in \operatorname{set}(m)$, as in the proof of Lemma 2.1, we have $x_{s} m=x_{t} w$ for some $w \in G\left(I^{k}\right), w<_{\text {revlex }} m, t \in\{1, \ldots, n\}$ and $s>t$. One may note that since $w \in G\left(I^{k}\right)$ and $x_{t} \mid m$, we must have $t \geq \min (\tilde{m})$ because otherwise, we get that $w=x_{s} m / x_{t} \operatorname{has} \operatorname{deg}(\bar{w})=k-1$, which is impossible.

In [7], J. Herzog and Y. Takayama defined the decomposition function of a monomial ideal with linear quotients. We recall their definition.

Definition 2.4 ([7, Definition 1.9]). Let $I \subset S$ be a monomial ideal with linear quotients with respect to the sequence of minimal monomial generators $u_{1}, \ldots, u_{m}$ and set $I_{j}=\left(u_{1}, \ldots, u_{j}\right)$ for $j=1, \ldots, m$. Let $M(I)$ be the set of all monomials in $I$. The map $g: M(I) \rightarrow G(I)$ defined as $g(u)=u_{j}$, where $j$ is the smallest number such that $u \in I_{j}$, is called the decomposition function of $I$.

By using the above results, we may completely describe the decomposition function associated to the increasing reverse lexicographical order. Note that since $I$ is a lexsegment ideal with a linear resolution which is not a completely lexsegment, $I$ has linear quotients with respect to the increasing reverse lexicographical order. Moreover, $I^{k}$ has linear quotients for all $k \geq 1$ by $[6$, Corollary $3.9]$.

Proposition 2.5. Let $I=(\mathcal{L}(u, v)) \subset S$ be a lexsegment ideal with a linear resolution which is not a completely lexsegment ideal and $g: M\left(I^{k}\right) \rightarrow G\left(I^{k}\right)$ the decomposition function with respect to the increasing reverse lexicographical order. Then

$$
g\left(x_{s} m\right)= \begin{cases}x_{s} m / x_{\min (m)}, & x_{s} m / x_{\min (m)} \succeq v^{k}, \\ x_{s} m / x_{\min (\tilde{m})}, & x_{s} m / x_{\min (m)} \prec v^{k}\end{cases}
$$

for any $m \in G\left(I^{k}\right), s \in \operatorname{set}(m)$, and $m=\bar{m} \tilde{m}$ 
We divide the proof into a sequence of lemmas.

Lemma 2.6. Let $I=(\mathcal{L}(u, v)) \subset S$ be a lexsegment ideal with a linear resolution which is not a completely lexsegment ideal and $g: M\left(I^{k}\right) \rightarrow G\left(I^{k}\right)$ the decomposition function with respect to the increasing reverse lexicographical order. If $m \in G\left(I^{k}\right)$ and $s \in \operatorname{set}(m)$ such that $x_{s} m / x_{\min (m)} \succeq v^{k}$, then $g\left(x_{s} m\right)=x_{s} m / x_{\min (m)}$.

Proof. Let $m \in G\left(I^{k}\right)$ and $s \in \operatorname{set}(m)$. We need to show that $x_{s} m / x_{\min (m)} \in$ $G\left(I^{k}\right)$ and that

$$
\frac{x_{s} m}{x_{\min (m)}}=\min { }_{\text {revlex }}\left\{w \in G\left(I^{k}\right): w<_{\text {revlex }} m, x_{s} m \in I_{\leq_{\text {revlex }} w}^{k}\right\} .
$$

If $x_{s} m / x_{\min (m)}=v^{k}$, then it is obvious that $x_{s} m / x_{\min (m)} \in G\left(I^{k}\right)$. Let us assume that $x_{s} m / x_{\min (m)} \succ v^{k}$. By Lemma 2.1, we have $s>\min (m)$. Since $x_{s} m / x_{\min (m)} \succ v^{k}$, we have either $\operatorname{deg}\left(\overline{x_{s} m / x_{\min (m)}}\right)>\operatorname{deg}\left(\overline{v^{k}}\right)=k$ or $\operatorname{deg}\left(\overline{x_{s} m / x_{\min (m)}}\right)=\operatorname{deg}\left(\overline{v^{k}}\right)=k$ and $x_{s} m / x_{\min (m)}>_{\text {lex }} v^{k}$.

In order to show that $x_{s} m / x_{\min (m)} \in G\left(I^{k}\right)$, we split the proof into two cases:

Case I: We assume that $\operatorname{deg}\left(\overline{x_{s} m / x_{\min (m)}}\right)>\operatorname{deg}\left(\overline{v^{k}}\right)=k$. Since $m \in$ $G\left(I^{k}\right)$, there exist $m_{1}, \ldots, m_{k} \in \mathcal{L}(u, v)$ such that $m=m_{1} \cdots m_{k}$. Let $1 \leq i \leq$ $k$ be such that $\min (m)=\min \left(m_{i}\right)$. Then

$$
\frac{x_{s} m}{x_{\min (m)}}=x_{s} m_{1} \cdots m_{i-1} \frac{m_{i}}{x_{\min \left(m_{i}\right)}} m_{i+1} \cdots m_{k} \geq v^{k} \text {. }
$$

If $x_{s} m_{i} / x_{\min \left(m_{i}\right)} \in \mathcal{L}(u, v)$, then we are done. Now let us assume that

$$
x_{s} m_{i} / x_{\min \left(m_{i}\right)} \notin \mathcal{L}(u, v),
$$

that is, $x_{s} m_{i} / x_{\min \left(m_{i}\right)}<_{\text {lex }} v=x_{l} x_{n}^{d-1}$ since $s>\min \left(m_{i}\right)=\min (m)$. In particular, $\operatorname{supp}\left(x_{s} m_{i} / x_{\min \left(m_{i}\right)}\right) \subseteq\{l+1, \ldots, n\}$ and $s \geq l+1$. Since

$$
\operatorname{deg}\left(\overline{x_{s} m / x_{\min (m)}}\right)>k,
$$

there exist $1 \leq j, r \leq l$ and $1 \leq \alpha \leq k$ such that $x_{j} x_{r} \mid m_{\alpha}$. In particular, we must have $j, r \geq 2$ by using the form of the monomials $u$ and $v$. Then

$$
\frac{x_{s} m}{x_{\min (m)}}=m_{1} \cdots \frac{x_{s} m_{\alpha}}{x_{j}} \cdots \frac{x_{j} m_{i}}{x_{\min \left(m_{i}\right)}} \cdots m_{k} \geq_{l e x} v^{k},
$$

where $v \leq_{\text {lex }} x_{j} m_{i} / x_{\min \left(m_{i}\right)} \leq_{\text {lex }} m_{i} \leq_{\text {lex }} u$ and $v \leq_{\text {lex }} x_{s} m_{\alpha} / x_{j}<_{\text {lex }} m_{\alpha} \leq_{\text {lex }}$ $u$. This implies $x_{s} m / x_{\min (m)} \in G\left(I^{k}\right)$.

Case II: We assume that $\operatorname{deg}\left(\overline{x_{s} m / x_{\min (m)}}\right)=\operatorname{deg}\left(\overline{v^{k}}\right)=k$. Therefore, we must have $x_{s} m / x_{\min (m)}>_{\text {lex }} v^{k}$. Since $\operatorname{deg}\left(\overline{x_{s} m / x_{\min (m)}}\right)=k$, we have either $s \leq l$ or $s>l$ and $\operatorname{deg}(\bar{m})=k+1$.

Since $m \in G\left(I^{k}\right)$, there exist $m_{1}, \ldots, m_{k} \in \mathcal{L}(u, v)$ such that $m=m_{1} \cdots m_{k}$. Let $1 \leq i \leq n$ be such that $\min (m)=\min \left(m_{i}\right)$.

If $s \leq l$, then since $m=m_{1} \cdots m_{k}$ and using the above notations, we get 


$$
\frac{x_{s} m}{x_{\min (m)}}=m_{1} \cdots m_{i-1} \frac{x_{s} m_{i}}{x_{\min \left(m_{i}\right)}} m_{i+1} \cdots m_{k} \geq v^{k} \in G\left(I^{k}\right)
$$

because $\min (m)=\min \left(m_{i}\right)<s \leq l$.

Analysis similar to that in the Case I shows that if $s>l$, then $x_{s} m / x_{\min (m)}$ $\in G\left(I^{k}\right)$.

We need to prove that

$$
\frac{x_{s} m}{x_{\min (m)}}=\min { }_{\text {revlex }}\left\{w \in G\left(I^{k}\right): w<_{\text {revlex }} m, x_{s} m \in I_{\leq_{\text {revlex }} w}^{k}\right\} .
$$

Let $w \in G\left(I^{k}\right)$ be such that $w<_{\text {revlex }} m$ and $x_{s} m \in I_{\leq_{\text {revlex }} w}^{k}$. Then there exists $w_{1} \in G\left(I^{k}\right), w_{1} \leq_{\text {revlex }} w$, such that $x_{s} m=x_{t} w_{1}$ for some $t, 1 \leq t \leq n$. As $m \neq w_{1}$, we have $s \neq t$, and hence $x_{t} \mid m$. Thus $t \geq \min (m)$. Therefore,

$$
w \geq_{\text {revlex }} w_{1}=\frac{x_{s} m}{x_{t}} \geq_{\text {revlex }} \frac{x_{s} m}{x_{\min (m)}}
$$

as desired.

Lemma 2.7. Let $I=(\mathcal{L}(u, v)) \subset S$ be a lexsegment ideal with a linear resolution which is not a completely lexsegment ideal and $g: M\left(I^{k}\right) \rightarrow G\left(I^{k}\right)$ the decomposition function with respect to the increasing reverse lexicographical order. If $m \in G\left(I^{k}\right)$ and $s \in \operatorname{set}(m)$ such that $x_{s} m / x_{\min (m)} \prec v^{k}$, then $g\left(x_{s} m\right)=x_{s} m / x_{\min (\tilde{m})}$.

Proof. As in the proof of Lemma 2.3, we see that $\operatorname{deg}\left(\overline{x_{s} m / x_{\min (m)}}\right)<k$ which implies that $\operatorname{deg}(\bar{m})=k$. By Lemma 2.3, we have $s>\min (\tilde{m})>l$.

Firstly, we prove that $x_{s} m / x_{\min (\tilde{m})} \in G\left(I^{k}\right)$. Since $m \in G\left(I^{k}\right)$, there exist $m_{1}, \ldots, m_{k} \in \mathcal{L}(u, v)$ such that $m=m_{1} \cdots m_{k}$. Let $1 \leq i \leq k$ be such that $x_{\min (\tilde{m})} \mid m_{i}$. Then

$$
\frac{x_{s} m}{x_{\min (\tilde{m})}}=m_{1} \cdots m_{i-1} \frac{x_{s} m_{i}}{x_{\min \left(m_{i}^{\prime}\right)}} \cdots m_{k} \in G\left(I^{k}\right)
$$

since $x_{s} m_{i} / x_{\min \left(m_{i}^{\prime}\right)} \in \mathcal{L}(u, v)$ because $s>\min (\tilde{m}) \geq l+1$.

Next, we prove that

$$
\frac{x_{s} m}{x_{\min (\tilde{m})}}=\min { }_{\text {revlex }}\left\{w \in G\left(I^{k}\right): w<_{\text {revlex }} m, x_{s} m \in I_{\leq_{\text {revlex }} w}^{k}\right\} .
$$

Let $w \in G\left(I^{k}\right)$ be such that $w<_{\text {revlex }} m$ and $x_{s} m \in I_{\leq_{\text {revlex }} w}^{k}$ which implies that there exists $w_{1} \in G\left(I^{k}\right), w_{1} \leq_{\text {revlex }} w$ such that $x_{s} m=x_{t} w_{1}$ for some $t$, $1 \leq t \leq n$. Obviously, $m \neq w_{1}$ implies $s \neq t$. Hence we must have $x_{t} \mid m$. In particular, $t \geq \min (m)$. Since $\operatorname{deg}(\bar{m})=k, s>\min (\tilde{m})>l$, and $w \in G\left(I^{k}\right)$, we must have that $\operatorname{deg}(\bar{w})=k$ which implies that $t \geq \min (\tilde{m})$ since $x_{t} \mid m$. Therefore, $w_{1}=x_{s} m / x_{t} \geq_{\text {revlex }} x_{s} m / x_{\min (\tilde{m})}$, which ends the proof.

Let $I$ be a monomial ideal with linear quotients. We say that the decomposition function $g: M(I) \rightarrow G(I)$ associated to the corresponding order of monomials is regular if $\operatorname{set}\left(g\left(x_{s} u\right)\right) \subseteq \operatorname{set}(u)$ for all $s \in \operatorname{set}(u)$ and $u \in G(I)$. In 
the sequel, we show that for the powers of lexsegment ideals $I$ with a linear resolution which are not completely lexsegment, the decomposition function $g: M\left(I^{k}\right) \rightarrow G\left(I^{k}\right)$ associated to the increasing reverse lexicographical order of the generators from $G\left(I^{k}\right)$ is regular.

Theorem 2.8. Let $I=(\mathcal{L}(u, v)) \subseteq S$ be a lexsegment ideal generated in degree $d>1$ with a linear resolution which is not a completely lexsegment ideal. Then the decomposition function $g: M\left(I^{k}\right) \rightarrow G\left(I^{k}\right)$ associated to the increasing reverse lexicographical order of the generators from $G\left(I^{k}\right)$ is regular.

For simplicity, we divide the proof into a sequence of lemmas.

Lemma 2.9. Let $I=(\mathcal{L}(u, v)) \subset S$ be a lexsegment ideal with a linear resolution which is not a completely lexsegment ideal and $g: M\left(I^{k}\right) \rightarrow G\left(I^{k}\right)$ the decomposition function with respect to the increasing reverse lexicographical order. Let $m \in G\left(I^{k}\right)$ and $s \in \operatorname{set}(m)$ be such that $x_{s} m / x_{\min (m)} \succ v^{k}$ and let $t \in \operatorname{set}\left(g\left(x_{s} m\right)\right)$. Then $t \in \operatorname{set}(m)$.

Proof. By Lemma 2.1, we have $s>\min (m)$. By hypothesis, $x_{s} m / x_{\min (m)} \succ v^{k}$. Therefore, by Lemma 2.5, we have $g\left(x_{s} m\right)=x_{s} m / x_{\min (m)}=w_{1}$. Since $t \in$ $\operatorname{set}\left(w_{1}\right)$, we get $x_{t} w_{1} \in I_{<\text {revlex }}^{k} w_{1}$. Hence there exist $w \in G\left(I^{k}\right), w<_{\text {revlex }} w_{1}$, and $1 \leq j \leq n$ such that $x_{t} w_{1}=x_{j} w$, that is,

$$
x_{t} x_{s} m=x_{j} x_{\min (m)} w .
$$

One may note that $j \neq t$ (otherwise, $w=w_{1}$, a contradiction), and hence $x_{j} \mid x_{s} m$. Since $t \in \operatorname{set}\left(w_{1}\right)$ and by using Lemma 2.1, we obtain that $t>$ $\min \left(w_{1}\right) \geq \min (m)$.

If $j=s$, then $x_{t} m=x_{\min (m)} w$ and $t \in \operatorname{set}(m)$.

Let us assume that $j \neq s$. We show that $x_{\min (m)} w / x_{s} \in G\left(I^{k}\right)$. We write $m=m_{1} \cdots m_{k}$ with $m_{1}, \ldots, m_{k} \in \mathcal{L}(u, v)$. Let $1 \leq i \leq k$ be such that $x_{j} \mid m_{i}$. Now, the fact that $w<_{\text {revlex }} w_{1}$ implies that $x_{\min (m)} w<_{\text {revlex }}$ $x_{\min (m)} w_{1}=x_{s} m$. Therefore, $x_{\min (m)} w / x_{s}<_{\text {revlex }} m$ and, taking into account that $x_{\min (m)} w / x_{s}=x_{t} m / x_{j}$, we get $x_{t} m / x_{j}<_{\text {revlex }} m$, that is, $t>j$.

Firstly, let us assume that $\operatorname{deg}(\bar{m})>k$ and let $1 \leq i \leq k$ such that $x_{j} \mid m_{i}$. Then

$$
\frac{x_{t} m}{x_{j}}=m_{1} \cdots \frac{x_{t} m_{i}}{x_{j}} \cdots m_{k} .
$$

If $x_{t} m_{i} / x_{j} \in \mathcal{L}(u, v)$, then we are done. Thus let us assume that $x_{t} m_{i} / x_{j} \notin$ $\mathcal{L}(u, v)$, which implies that $x_{t} m_{i} / x_{j}<_{\text {lex }} v$ since $x_{t} m_{i} / x_{j}<_{\text {lex }} m_{i} \leq_{\text {lex }} u$ $(t>j)$. In this case, there exist $1 \leq p \leq k$ and $1 \leq \alpha \leq l$ such that $\operatorname{deg}\left(\overline{m_{p}}\right) \geq 2$ and $x_{\alpha} \mid m_{p}$. In particular, we must have $\alpha \geq 2$ (by using the form of the monomials $u$ and $v$ ). In this case,

$$
\frac{x_{t} m}{x_{j}}=m_{1} \cdots \frac{x_{\alpha} m_{i}}{x_{j}} \cdots \frac{x_{t} m_{p}}{x_{\alpha}} \cdots m_{k},
$$

which implies $x_{t} m / x_{j} \in G\left(I^{k}\right)$ since $v \leq_{\text {lex }} x_{\alpha} x_{n}^{d-1} \leq_{\text {lex }} x_{\alpha} m_{i} / x_{j}<_{\text {lex }} u$ and $v \leq_{\text {lex }} x_{\alpha} x_{n}^{d-1} \leq_{\text {lex }} x_{t} m_{p} / x_{\alpha}<_{\text {lex }} u$. 
Let us assume that $\operatorname{deg}(\bar{m})=k$. Since $j<t$, we get $x_{t} m / x_{j}<_{\text {lex }} m$. If $\operatorname{deg}\left(\overline{x_{t} m / x_{j}}\right)=k$, then we obviously have $x_{t} m / x_{j} \in G\left(I^{k}\right)$. We assume that $\operatorname{deg}\left(\overline{x_{t} m / x_{j}}\right)=k-1$, that is, $j \leq l$ and $t>l$. We also have $\min (m) \leq l$. Hence $\operatorname{deg}(\bar{m})=k$ and the equality $x_{t} x_{s} m=x_{j} x_{\min (m)} w$ imply

$$
k \leq \operatorname{deg}(\bar{w})=\operatorname{deg}(\bar{m})+\nu_{1}\left(x_{t} x_{s}\right)+\cdots+\nu_{l}\left(x_{t} x_{s}\right)-2,
$$

which yields $\nu_{1}\left(x_{t} x_{s}\right)+\cdots+\nu_{l}\left(x_{t} x_{s}\right)=2$, that is, $t, s \leq l$, a contradiction.

We proved that $x_{\min (m)} w / x_{s}<_{\text {revlex }} m$ and that $x_{\min (m)} w / x_{s} \in G\left(I^{k}\right)$. Hence $t \in \operatorname{set}(m)$.

Lemma 2.10. Let $I=(\mathcal{L}(u, v)) \subset S$ be a lexsegment ideal with a linear resolution which is not a completely lexsegment ideal and $g: M\left(I^{k}\right) \rightarrow G\left(I^{k}\right)$ the decomposition function with respect to the increasing reverse lexicographical order. Let $m \in G\left(I^{k}\right)$ and $s \in \operatorname{set}(m)$ be such that $x_{s} m / x_{\min (m)} \prec v^{k}$ and let $t \in \operatorname{set}\left(g\left(x_{s} m\right)\right)$. Then $t \in \operatorname{set}(m)$.

Proof. According to Proposition 2.5, we have $g\left(x_{s} m\right)=x_{s} m / x_{\min (\tilde{m})}=\omega$. Since $t \in \operatorname{set}(\omega)$, we get $x_{t} \omega \in I_{<_{\text {revlex }} \omega}^{k}$. Hence as in the proof of Lemma 2.1, $x_{t} \omega=x_{j} w$ for some $w \in G\left(I^{k}\right), w<_{\text {revlex }} \omega$, and $t>j \geq \min (\omega)$. Therefore, we get that

$$
x_{t} x_{s} m=x_{j} x_{\min (\tilde{m})} w .
$$

Also, one may note that the only possible case is that in which

$$
\operatorname{deg}\left(\overline{x_{s} m / x_{\min (m)}}\right)<k \text {. }
$$

Indeed, since $x_{s} m / x_{\min (m)} \prec v^{k}$, we have either $\operatorname{deg}\left(\overline{x_{s} m / x_{\min (m)}}\right)<\operatorname{deg}\left(\overline{v^{k}}\right)=$ $k$ or $\operatorname{deg}\left(\overline{x_{s} m / x_{\min (m)}}\right)=\operatorname{deg}\left(\overline{v^{k}}\right)$ and $x_{s} m / x_{\min (m)}<_{l e x} v^{k}=x_{l}^{k} x_{n}^{k(d-1)}$. If we assume that $\operatorname{deg}\left(\overline{x_{s} m / x_{\min (m)}}\right)=\operatorname{deg}\left(\overline{v^{k}}\right)$ and that $x_{s} m / x_{\min (m)}<_{l e x} v^{k}=$ $x_{l}^{k} x_{n}^{k(d-1)}$, then we have $x_{s} m / x_{\min (m)} \leq_{l e x} x_{l}^{k-1} x_{l+1}^{k(d-1)+1}$ and $\operatorname{deg}\left(\overline{x_{s} m / x_{\min (m)}}\right)$ $<k$, a contradiction. By Lemma 2.3, we have $s>\min (\tilde{m})$. In particular, $\operatorname{deg}(\bar{m})=\operatorname{deg}(\bar{\omega})=k$. Moreover, $\operatorname{deg}(\bar{w}) \leq \operatorname{deg}(\bar{\omega})$ implies $\operatorname{deg}(\bar{w})=k$ since $w \in G\left(I^{k}\right)$.

If $j=s$, then $x_{t} m=x_{\min (\tilde{m})} w$ and $t \in \operatorname{set}(m)$.

We assume now that $j \neq s$. By the equality $(*)$, we also have $x_{\min (\tilde{m})} w / x_{s}=$ $x_{t} m / x_{j}$. If $\bar{w}=x_{1}^{k}$, then $x_{1}^{k} \mid m$ since $s>\min (\tilde{m})>l$ and $t>j \geq \min (\omega)=$ $\min (m)$. Now, equality $(*)$ gives $j>l$. Therefore, $x_{t} m / x_{j} \in G\left(I^{k}\right)$, and thus $x_{\min (\tilde{m})} w / x_{s} \in G\left(I^{k}\right)$.

Let us consider the case when $\bar{w} \neq x_{1}^{k}$ and let $w=w_{1} \cdots w_{k}$. Therefore, there exists $1 \leq i \leq k$ such that $x_{1} \nmid w_{i}$. If $x_{s} \mid w_{i}$, then $v \leq_{\text {lex }} x_{\min (\tilde{m})} w_{i} / x_{s}<_{\text {lex }}$ $w_{i} \leq_{l e x} u$ and $x_{\min (\tilde{m})} w / x_{s} \in G\left(I^{k}\right)$. If $x_{s} \nmid w_{i}$, then let $1 \leq j \leq k$ be such that $x_{s} \mid w_{j}$ and

$$
\frac{x_{\min (\tilde{m})} w}{x_{s}}=w_{1} \cdots \frac{x_{\max \left(w_{i}\right)} w_{j}}{x_{s}} \cdots \frac{x_{\min (\tilde{m})} w_{i}}{x_{\max \left(w_{i}\right)}} \cdots w_{k} .
$$


Since $\operatorname{deg}(\bar{w})=k$, we must have $\max \left(w_{i}\right)>l$. Thus $v \leq_{l e x} x_{\max \left(w_{i}\right)} w_{j} / x_{s} \leq_{u}$ and $v \leq_{l e x} x_{\min (\tilde{m})} w_{i} / x_{\max \left(w_{i}\right)} \leq_{l e x} u$, and therefore, $x_{\min (\tilde{m})} w / x_{s} \in G\left(I^{k}\right)$. Moreover, $x_{\min (\tilde{m})} w / x_{s}=x_{t} m / x_{j}<_{\text {revlex }} m$. Hence $t \in \operatorname{set}(m)$.

Lemma 2.11. Let $I=(\mathcal{L}(u, v)) \subset S$ be a lexsegment ideal with a linear resolution which is not a completely lexsegment ideal and $g: M\left(I^{k}\right) \rightarrow G\left(I^{k}\right)$ the decomposition function with respect to the increasing reverse lexicographical order. Let $m \in G\left(I^{k}\right)$ and $s \in \operatorname{set}(m)$ be such that $x_{s} m / x_{\min (m)}=v^{k}$ and let $t \in \operatorname{set}\left(g\left(x_{s} m\right)\right)$. Then $t \in \operatorname{set}(m)$.

Proof. In this case, one can easily see that we can have either $s \leq l$, which implies, in fact, that $s=l$, or $s>l$ and $\operatorname{deg}(\bar{m})=k+1$.

By Proposition 2.5, we have $g\left(x_{s} m\right)=x_{s} m / x_{\min (m)}=v^{k}=w_{1}$. Since $t \in \operatorname{set}\left(w_{1}\right)$, we get $x_{t} w_{1} \in I_{<_{\text {revlex }} w_{1}}^{k}$. Hence as in the proof of Lemma 2.1, $x_{t} w_{1}=x_{j} w$ for some $w \in G\left(I^{k}\right), w<_{\text {revlex }} w_{1}$, and $t>j \geq \min \left(w_{1}\right)=l$. Note that $\operatorname{deg}(\bar{w}) \leq \operatorname{deg}\left(\overline{w_{1}}\right)=k$, which implies $\operatorname{deg}(\bar{w})=k$. Therefore, we get that

$$
x_{t} x_{s} m=x_{j} x_{\min (m)} w .
$$

If $j=s$, then $x_{t} m=x_{n} w$ and $t \in \operatorname{set}(m)$. Therefore, we assume that $j \neq s$.

The case when $s=l$ is impossible. Indeed, if $s=l$, then we must have $j>l$ since $j \geq \min \left(w_{1}\right)=l$ and $j \neq s$. Thus $j=n$ since $x_{j} \mid w_{1}=v^{k}$. But this is a contradiction since $t \neq j$.

If $s>l$, then $s=n$. In this case, $\operatorname{deg}(\bar{m})=k+1$, which implies that $\operatorname{deg}(\bar{w})=k$ and $l<j<n$. Therefore, $x_{j} w / x_{s} \in G\left(I^{k}\right)$. Thus $x_{t} m=$ $x_{n}\left(x_{j} w / x_{s}\right)$ and $t \in \operatorname{set}(m)$.

By using the decomposition function, one may completely describe the resolution as shown by J. Herzog and Y. Takayama [7].

Lemma 2.12 ([7, Lemma 1.5]). Suppose $\operatorname{deg} u_{1} \leq \operatorname{deg} u_{2} \leq \cdots \leq \operatorname{deg} u_{m}$. Then the iterated mapping cone $\mathbb{F}$ derived from the sequence $u_{1}, \ldots, u_{m}$ is a minimal graded free resolution of $S / I$, and for all $i>0$, the symbols

$$
f(\sigma ; u) \text { with } u \in G(I), \sigma \subset \operatorname{set}(u),|\sigma|=i-1
$$

form a homogeneous basis of the $S$-module $F_{i}$. Moreover, $\operatorname{deg}(f(\sigma ; u))=$ $|\sigma|+\operatorname{deg}(u)$.

Theorem 2.13 ([7, Theorem 1.12]). Let I be a monomial ideal of $S$ with linear quotients and $\mathbb{F}$. the graded minimal free resolution of $S / I$. Suppose that the decomposition function $g: M(I) \rightarrow G(I)$ is regular. Then the chain map $\partial$ of $\mathbb{F} \bullet$ is given by

$\partial(f(\sigma ; u))=-\sum_{s \in \sigma}(-1)^{\alpha(\sigma ; s)} x_{s} f(\sigma \backslash s ; u)+\sum_{s \in \sigma}(-1)^{\alpha(\sigma ; s)} \frac{x_{s} u}{g\left(x_{s} u\right)} f\left(\sigma \backslash s ; g\left(x_{s} u\right)\right)$

if $\sigma \neq \emptyset$, and

$$
\partial(f(\emptyset ; u))=u
$$

otherwise. Here, $\alpha(\sigma ; s)=|\{t \in \sigma \mid t<s\}|$. 
In our specific context, we get the following.

Corollary 2.14. Let $I=(\mathcal{L}(u, v)) \subset S$ be a lexsegment ideal with linear quotients with respect to increasing reverse lexicographical order which is not a completely lexsegment ideal and $\mathbb{F} \bullet$ the graded minimal free resolution of $S / I^{k}$. Then the chain map of $\mathbb{F} \bullet$ is given by

$$
\begin{aligned}
\partial(f(\sigma ; w))= & \sum_{\substack{s \in \sigma: \\
x_{s} w / x_{\min (w)} \succeq v^{k}}}(-1)^{\alpha(\sigma ; s)} x_{\min (w)} f\left(\sigma \backslash s ; \frac{x_{s} w}{x_{\min (w)}}\right) \\
& +\sum_{\substack{s \in \sigma: \\
x_{s} w / x_{\min (w)} \prec v^{k}}}(-1)^{\alpha(\sigma ; s)} x_{\min (\tilde{w})} f\left(\sigma \backslash s ; \frac{x_{s} w}{x_{\min (\tilde{w})}}\right) \\
& -\sum_{s \in \sigma}(-1)^{\alpha(\sigma ; s)} x_{s} f(\sigma \backslash s ; w)
\end{aligned}
$$

if $\sigma \neq \emptyset$, and

$$
\partial(f(\emptyset ; w))=w
$$

otherwise. For convenience, we set $f(\sigma ; w)=0$ if $\sigma \nsubseteq \operatorname{set} w$.

\section{An example}

Let $u=x_{1} x_{4}$ and $v=x_{2} x_{5}$ be monomials in the polynomial ring $S=$ $k\left[x_{1}, x_{2}, x_{3}, x_{4}, x_{5}\right]$. Then

$$
\mathcal{L}(u, v)=\left\{x_{1} x_{4}, x_{1} x_{5}, x_{2}^{2}, x_{2} x_{3}, x_{2} x_{4}, x_{2} x_{5}\right\} .
$$

The ideal $I=(\mathcal{L}(u, v))$ is a lexsegment ideal which is not completely lexsegment. According to [6, Corollary 3.9], the ideal $I^{2}$ has linear quotients with respect to the following order of the generators: $u_{1}=x_{2}^{2} x_{5}^{2}, u_{2}=x_{1} x_{2} x_{5}^{2}$, $u_{3}=x_{1}^{2} x_{5}^{2}, u_{4}=x_{2}^{2} x_{4} x_{5}, u_{5}=x_{1} x_{2} x_{4} x_{5}, u_{6}=x_{1}^{2} x_{4} x_{5}, u_{7}=x_{2}^{2} x_{3} x_{5}, u_{8}=$ $x_{1} x_{2} x_{3} x_{5}, u_{9}=x_{2}^{3} x_{5}, u_{10}=x_{1} x_{2}^{2} x_{5}, u_{11}=x_{2}^{2} x_{4}^{2}, u_{12}=x_{1} x_{2} x_{4}^{2}, u_{13}=x_{1}^{2} x_{4}^{2}$, $u_{14}=x_{2}^{2} x_{3} x_{4}, u_{15}=x_{1} x_{2} x_{3} x_{4}, u_{16}=x_{2}^{3} x_{4}, u_{17}=x_{1} x_{2}^{2} x_{4}, u_{18}=x_{2}^{2} x_{3}^{2}, u_{19}=$ $x_{2}^{3} x_{3}, u_{20}=x_{2}^{4}$. We have $\operatorname{set}\left(u_{1}\right)=\emptyset, \operatorname{set}\left(u_{2}\right)=\{2\}, \operatorname{set}\left(u_{3}\right)=\{2\}, \operatorname{set}\left(u_{4}\right)=$ $\{5\}, \operatorname{set}\left(u_{5}\right)=\{2,5\}, \operatorname{set}\left(u_{6}\right)=\{2,5\}, \operatorname{set}\left(u_{7}\right)=\{4,5\}, \operatorname{set}\left(u_{8}\right)=\{2,4,5\}$, $\operatorname{set}\left(u_{9}\right)=\{3,4,5\}, \operatorname{set}\left(u_{10}\right)=\{2,3,4,5\}, \operatorname{set}\left(u_{11}\right)=\{5\}, \operatorname{set}\left(u_{12}\right)=\{2,5\}$, $\operatorname{set}\left(u_{13}\right)=\{2,5\}, \operatorname{set}\left(u_{14}\right)=\{4,5\}, \operatorname{set}\left(u_{15}\right)=\{2,4,5\}, \operatorname{set}\left(u_{16}\right)=\{3,4,5\}$, $\operatorname{set}\left(u_{17}\right)=\{2,3,4,5\}, \operatorname{set}\left(u_{18}\right)=\{4,5\}, \operatorname{set}\left(u_{19}\right)=\{3,4,5\}, \operatorname{set}\left(u_{20}\right)=\{3,4,5\}$. Note that in this case, the integer $l$ that we fix for defining the order $\prec$ is $l=2$. Let $\mathbb{F} \bullet$ be the minimal graded free resolution of $S / I$.

Since $\max \{|\operatorname{set}(w)|: w \in \mathcal{L}(u, v)\}=4$, we have $F_{i}=0$ for all $i \geq 6$.

A basis for the $S$-module $F_{1}$ is $\left\{f\left(\emptyset ; u_{1}\right), \ldots, f\left(\emptyset ; u_{20}\right)\right\}$.

A basis for the $S$-module $F_{2}$ is $\left\{f\left(\{i\} ; u_{j}\right): i \in \operatorname{set}\left(u_{j}\right), 1 \leq j \leq 20\right\}$ having cardinality 44.

A basis for the $S$-module $F_{3}$ is $\left.\left\{f\left(\{i, j\} ; u_{k}\right),\{i, j\} \subseteq \operatorname{set}\left(u_{k}\right), 1 \leq k \leq 20\right\}\right\}$ having cardinality 37 . 
A basis for the $S$-module $F_{4}$ is

$\left\{f\left(\{2,4,5\} ; u_{8}\right), f\left(\{3,4,5\} ; u_{9}\right), f\left(\{2,3,4\} ; u_{10}\right), f\left(\{2,3,5\} ; u_{10}\right)\right.$, $f\left(\{2,4,5\} ; u_{10}\right), f\left(\{3,4,5\} ; u_{10}\right), f\left(\{2,4,5\} ; u_{15}\right), f\left(\{3,4,5\} ; u_{16}\right)$, $\left.f\left(\{2,3,4\} ; u_{17}\right), f\left(\{2,3,5\} ; u_{17}\right), f\left(\{2,4,5\} ; u_{17}\right), f\left(\{3,4,5\} ; u_{17}\right)\right\}$.

A basis for the $S$-module $F_{5}$ is $\left\{f\left(\{2,3,4,5\} ; u_{10}\right), f\left(\{2,3,4,5\} ; u_{17}\right)\right\}$.

We have the minimal graded free resolution $\mathbb{F}_{\bullet}$ :

$0 \rightarrow S(-8)^{2} \stackrel{\partial_{4}}{\rightarrow} S(-7)^{14} \stackrel{\partial_{3}}{\rightarrow} S(-6)^{37} \stackrel{\partial_{2}}{\rightarrow} S(-5)^{44} \stackrel{\partial_{1}}{\rightarrow} S(-4)^{20} \stackrel{\partial_{0}}{\rightarrow} S \rightarrow S / I \rightarrow 0$.

We will determine only the differentials $\partial_{0}$ and $\partial_{4}$.

It is easily seen that the differential $\partial_{0}$ is given by

$$
\partial_{0}\left(f\left(\emptyset ; u_{i}\right)\right)=u_{i} \text { for } 1 \leq i \leq 20 \text {. }
$$

We determine now the differential $\partial_{4}$. For $\partial_{4}\left(f\left(\{2,3,4,5\} ; u_{10}\right)\right)$, one may note that $x_{s} u_{10} / x_{\min \left(u_{10}\right)} \succeq v^{2}$ for all $s \in \operatorname{set}\left(u_{10}\right)$. Therefore,

$$
\begin{aligned}
\partial_{4}\left(f\left(\{2,3,4,5\} ; u_{10}\right)\right)= & x_{1} f\left(\{3,4,5\} ; u_{9}\right)-x_{1} f\left(\{2,4,5\} ; u_{7}\right) \\
& +x_{1} f\left(\{2,3,5\} ; u_{4}\right)-x_{1} f\left(\{2,3,4\} ; u_{3}\right) \\
& -x_{2} f\left(\{3,4,5\} ; u_{10}\right)+x_{3} f\left(\{2,4,5\} ; u_{10}\right) \\
& -x_{4} f\left(\{2,3,5\} ; u_{10}\right)+x_{5} f\left(\{2,3,4\} ; u_{10}\right) \\
= & x_{1} f\left(\{3,4,5\} ; u_{9}\right)-x_{2} f\left(\{3,4,5\} ; u_{10}\right) \\
& +x_{3} f\left(\{2,4,5\} ; u_{10}\right)-x_{4} f\left(\{2,3,5\} ; u_{10}\right) \\
& +x_{5} f\left(\{2,3,4\} ; u_{10}\right)
\end{aligned}
$$

since $\{2,4,5\} \nsubseteq \operatorname{set}\left(u_{7}\right),\{2,3,5\} \nsubseteq \operatorname{set}\left(u_{4}\right)$, and $\{2,3,4\} \nsubseteq \operatorname{set}\left(u_{3}\right)$.

For $\partial_{4}\left(f\left(\{2,3,4,5\} ; u_{17}\right)\right)$, one may note that $x_{s} u_{17} / x_{\min \left(u_{17}\right)} \succeq v^{2}$ for all $s \in \operatorname{set}\left(u_{17}\right)$. Therefore,

$$
\begin{aligned}
\partial_{4}\left(f\left(\{2,3,4,5\} ; u_{17}\right)\right)= & x_{1} f\left(\{3,4,5\} ; u_{16}\right)-x_{1} f\left(\{2,4,5\} ; u_{14}\right) \\
& +x_{1} f\left(\{2,3,5\} ; u_{11}\right)-x_{1} f\left(\{2,3,4\} ; u_{4}\right) \\
& -x_{2} f\left(\{3,4,5\} ; u_{17}\right)+x_{3} f\left(\{2,4,5\} ; u_{17}\right) \\
& -x_{4} f\left(\{2,3,5\} ; u_{17}\right)+x_{5} f\left(\{2,3,4\} ; u_{17}\right) \\
= & x_{1} f\left(\{3,4,5\} ; u_{16}\right)-x_{2} f\left(\{3,4,5\} ; u_{17}\right) \\
& +x_{3} f\left(\{2,4,5\} ; u_{17}\right)-x_{4} f\left(\{2,3,5\} ; u_{17}\right) \\
& +x_{5} f\left(\{2,3,4\} ; u_{17}\right)
\end{aligned}
$$


since $\{2,4,5\} \nsubseteq \operatorname{set}\left(u_{14}\right),\{2,3,5\} \nsubseteq \operatorname{set}\left(u_{11}\right)$, and $\{2,3,4\} \nsubseteq \operatorname{set}\left(u_{4}\right)$.

$$
\left(\begin{array}{cc}
0 & 0 \\
x_{1} & 0 \\
x_{5} & 0 \\
-x_{4} & 0 \\
x_{3} & 0 \\
-x_{2} & 0 \\
0 & 0 \\
0 & x_{1} \\
0 & x_{5} \\
0 & -x_{4} \\
0 & x_{3} \\
0 & -x_{2}
\end{array}\right)
$$

\section{References}

[1] A. Aramova, E. De Negri, and J. Herzog, Lexsegment ideals with linear resolutions, Illinois J. Math. 42 (1998), no. 3, 509-523.

[2] A. Conca, Regularity jumps for powers of ideals, Proceedings Lisbon Conference on Commutative Algebra, Lisbon-Portugal, 2003.

[3] A. Conca and J. Herzog, Castelnuovo-Mumford regularity of products of ideals, Collect. Math. 54 (2003), no. 2, 137-152.

[4] E. De Negri and J. Herzog, Completely lexsegment ideals, Proc. Amer. Math. Soc. 126 (1998), no. 12, 3467-3473.

[5] V. Ene, A. Olteanu, and L. Sorrenti, Properties of lexsegment ideals, Osaka J. Math. 47 (2010), no. 1, 67-87.

[6] V. Ene and A. Olteanu, Powers of lexsegment ideals with linear resolutions, arXiv:1011.2157, to appear in Illinois J. Math.

[7] J. Herzog and Y. Takayama, Resolutions by mapping cones, Homology Homotopy Appl. 4 (2002), no. 2, 277-294.

[8] H. Hulett and H. M. Martin, Betti numbers of lex-segment ideals, J. Algebra 275 (2004), no. $2,629-638$.

[9] M. Ishaq, Lexsegment ideals are sequentially Cohen-Macaulay, ArXiv:1010.5615v2.

[10] A. Olteanu, Normally torsion-free lexsegment ideals, arXiv: 1010.1473v1, to appear in Alg. Coll.

[11] A. Olteanu, O. Olteanu, and L. Sorrenti, Gotzmann lexsegment ideals, Matematiche (Catania) 63 (2008), no. 2, 229-241.

Faculty of Mathematics and Computer Science OVIDIUS UNIVERSITY

Bd. Mamaia 124, 900527 Constanta, Romania

E-mail address: olteanuandageorgiana@gmail.com 